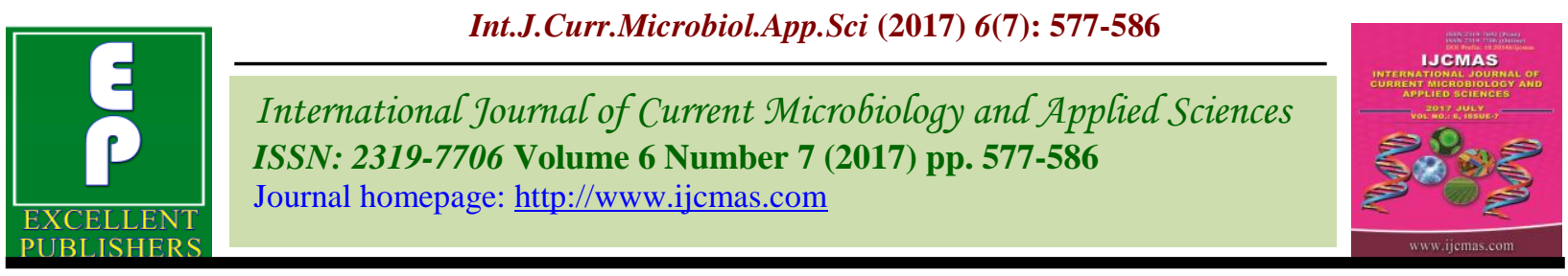

Original Research Article https://doi.org/10.20546/ijcmas.2017.607.070

\title{
Determination of maturity and Genetic Diversity in Mandarin (Citrus reticulata Blanco) Genotypes Based on Citrus Colour Index
}

\author{
Gurteg Singh $^{1^{*}}$, Phupinder Singh Aulakh ${ }^{2}$ and Harinder Singh Rattanpal ${ }^{1}$ \\ ${ }^{1}$ Department of Fruit Science, Punjab Agricultural University, Ludhiana-141 004, Punjab, India \\ ${ }^{2}$ Department of Horticulture, Punjab, India \\ *Corresponding author
}

\section{A B S T R A C T}

\begin{tabular}{|l|}
\hline Ke y w or d s \\
Citrus colour \\
index, Chromicity, \\
Diversity, Epicarp, \\
Genotype.
\end{tabular}

The genetic diversity among nineteen mandarin genotypes was assessed at Punjab Agricultural University Ludhiana based on fruit chromicity scores. Colour parameters of fruit epicarp, pulp and juice were determined with the help of Hunter Lab colorimetric system as per IPGRI descriptors. The mean value of epicarp CCI was significantly higher (11.91) in W. Murcott followed by Fremont (9.32) and Kinnow (8.54). However, the mean value of pulp CCI of different mandarin genotypes ranged from 4.28 to 11.30 with average value of 6.66. The pulp CCI was maximum (11.30) in Daisy which was statistically at par with Darjeeling (10.51). The data revealed that mean value of juice CCI was maximum (9.78) in Daisy which was statistically at par with W. Murcott (9.68), CRS-4 (9.14), Clone-11(9.03) and Kinnow (8.86). The clustering analysis showed that genotypes were grouped across the sub-groups based on their quantitative colorimetric values without clear evidence of their geographical distribution. The study concluded that W. Murcott, Fremont, Kinnow and Daisy are distinct genotypes having higher chromicity scores. Thus, this study will help citrus breeder to select distinct mandarin genotypes for targeting better fruit colour parameters in citrus improvement programme.

\section{Introduction}

Fruit colour is associated with freshness and is very critical for acceptance in the consumer's hands (Campbell et al., 2004). Both external and internal sensory parameters are important for the consumer. Easy pealing, pleasant flavor, low seediness and fragrance are most desirable internal quality parameters in mandarins (Jenks et al., 2011). Similarly, suitable fruit shape, deep peel colour and glossy surface are deciding external traits deciding attractiveness of fruits. The fruit colour is also an important consideration for post-harvest studies (Cubero et al., 2010) and colour change from green to yellowish is a most important maturity indices. The progressive citrus growers ensure the uniform quality in term of colour while packing the fruits in batches. Under sub-tropical conditions, the harvesting of the mandarins starts before they attain typical orange colour. The commercial growers have to give certain degreening treatments, depending upon their standard colour index at harvest (Vidal et al., 2013).

Colour is generally taken as an index of freshness, peelability and nutritional value by consumers (Haisman et al., 1975). This is a 
very complex trait which is controlled by chemical, biochemical and physicochemical mechanisms (Sinha et al., 2012). Moreover, fruit colour is governed by several other factors such as fruit maturity, tree nutrition, rootstock, agronomic practices, biotic and abiotic stresses (Ladanyia, 2010). Major colour pigments are cholorphyll (green), carotenoids (yellow, orange, and red deep orange), anthocyanins (red) and lucopene (red or pink). During October-November, the chlorophyll present in the peel is degraded and carotenes are freshly synthesized (Sinclair, 1984; Artes et al., 2002), which imparts lemon-yellow colour to the fruit. This attractive colour indicates the ripeness of fruit and stimulates perception of freshness in customer' mind (Hutchings, 2003).

No doubt, citrus breeding objectives may vary as per need of the region, but breeding for quality is one of the important objectives in most of regions of the world.

The selection of suitable parents in citrus breeding programme is very important. Recent trends and the major goals of the breeding program are focused on physical attributes like fruit colour, fruit size, easy peeling and seedlessness (Abouzar and Nafiseh, 2016). In conventional hybridization programme, breeder generally take one pigmented parent (Deng and XU, 2011).The citrus colour index (CCI) in the citrus industry is used to determine the harvesting date in different citrus genotypes (DOGV et al., 2006) Conventionally, colorimeter is also used for colour measurement with numerical figures, however, it is limited to the small region of fruit surface (Gardner, 2007)

Therefore, the study was planned with objectives to use high quality image acquisition system for exploring the true potential in the differentiation of genotypes on the basis of their colour and to determine the feasibility for the use of chromicity as fruit maturity index.

\section{Materials and Methods}

\section{Plant material}

Nineteen mandarin genotypes grafted on rough lemon rootstock planted at a spacing of $6 \times 3 \mathrm{~m}$ were used in this research work. The study was carried out at college orchard and PG lab of department of fruit science, Punjab Agricultural University, Ludhiana during 2013- 2015.

All the trees received recommended doses of fertilizers and other cultural practices during the course of these investigations. Randomly selected ten mature fruits were taken in each replication and the number of replications was three.

Peel colour parameters of fruit, viz. epicarp, pulp and juice were determined with the help of Hunter Lab colorimetric system as per IPGRI descriptors (IPGRI, 1999). The Hunter $\mathrm{L}$, $\mathrm{a}$ and $\mathrm{b}$ colour space is organized in a cube form. The ' $L$ ' axis runs from top to bottom. The maximum for ' $L$ ' is 100 , which would be a perfect reflecting diffuser. The minimum value for ' $L$ ' is zero, which would be black. The $a$ and $b$ axes have no specific numerical limits. Positive ' $a$ ' is red and negative ' $a$ ' is green. Positive ' $b$ ' is yellow and negative ' $b$ ' is blue (Hunter Lab, 2008). The colorimeter was calibrated using a standard calibration plate prior to each use. The colorimeter measured three variables: $a=$ green $/ \mathrm{red}, \mathrm{b}=$ blue/yellow and $\mathrm{L}=$ Luminosity. The value of citrus colour index was calculated as under.

Citrus Colour Index $=1000$ x a / (L x b)

Negative value of CCI means dark green/green colour. Value around zero means green-yellow colour (intermediate) and small 
positive value means yellow colour. High positive value means red-orange colour.

\section{Statistical analysis}

Quantitative data were analyzed using SAS (9.3 version) software and data were also subjected to un-weighted pair group method with arithmetic mean (UPGMA) by using DAR win software (Perrier and JacquemoudCollet, 2006).

\section{Results and Discussion}

\section{Epicarp colour parameters}

The mean epicarp colour lightness value (L) of different mandarin genotypes ranged from 50.64 to 61.68 with average value of 56.79 (Table 1). The mean maximum epicarp lightness (61.68) was reflected by genotype Kinnow which was statistically at par with Mudhkhed Seedless (59.79), Clone-11 (59.57), Daisy (59.36), N-51(58.87), Nagpur Seedless (58.78) and Nova (58.78) and it was significantly higher than all other genotypes. Mean redness and greenness value of different mandarin genotypes ranged from 14.34 to 31.58 with average value of 21.52 . The maximum mean redness and greenness value (31.58) was recorded in genotype W. Murcott and it was significantly higher than all other genotypes except Kinnow and Daisy. The mean maximum blueness and yellowness (61.18) was recorded in genotype Clone-11 and it was significantly higher than all other genotypes except Daisy, N-4, Nova, Kinnow and Nagpur. The CCI of fruit epicarp of different mandarin genotypes ranged from 4.51 to 11.91 with average value of 7.09 (Table 1). The data show that mean value of epicarp CCI was maximum (11.91) in W. Murcott followed by Fremont (9.32) and Kinnow (8.54) and it was significantly higher than all other genotypes under study.
The high positive value of CCI indicates the red orange colour of the fruit epicarp. Fruits from W. Murcott, Fremont and Kinnow genotypes had significantly higher value which clearly differentiated these fruit from all other genotypes. These genotypes are highly promising in term of red orange colour trait which is highly preferred traits in mandarin group. However, fruits from N-28 genotypes have low CCI value which indicates less promising orange colour of fruit epicarp.

\section{Pulp colour parameters}

The results (Table 2) revealed that fruit pulp colour lightness values among mandarin genotypes differed significantly. The mean value ranged from 40.84 to 57.89 with average value of 51.41. Maximum mean pulp lightness (57.89) was reflected by genotype $\mathrm{N}-28$ which was statistically at par with W. Murcott, Khasi, Nagpur, Nova, CRS-4, N-4, $\mathrm{N}-43$ and N-51. Mean redness and greenness values of among mandarin genotypes ranged from 5.14 to 10.79 with average value of 7.56. The mean maximum fruit pulp redness and greenness (10.79) was recorded in genotype Daisy and it was significantly higher than all other genotypes except Darjeeling (9.98). Fruit pulp mean blueness and yellowness value of different mandarin genotypes ranged from 15.43 to 29.08 with average value of 23.24. The maximum mean pulp blueness and yellowness (29.08) was recorded in genotype Kinnow and it was significantly higher than all other genotypes except Nova, Fremont, N-28, Khasi and W. Murcott. Maximum mean value of pulp CCI of different mandarin genotypes ranged from 4.28 to 11.30 with average value of 6.66 . The data show that mean value of pulp CCI was maximum (11.30) in Daisy which was statistically at par with Darjeeling (10.51) and it was significantly higher than all other genotypes. 
The high positive value of CCI in our study indicates the deep red orange colour of fruit pulp. Fruits from Daisy and Darjiling genotypes have significantly higher CCI values which differentiate these genotypes from all other fruits under investigation. The data clearly show that these genotypes are highly promising in term of pulp colour (deep red orange). However, fruits from N-51, N28, Nagpur, Nova, Khasi, Fremomt, N-43 and Mudhkhed Seedless genotypes have low CCI value indicating light orange colour of fruit pulp.

\section{Juice colour parameters}

It is evident from the data in table 3 that juice colour lightness values differed significantly among different genotypes. The mean juice colour lightness value of different mandarin genotypes ranged from 31.00 to 37.43 with average value of 35.19. Significantly higher mean juice lightness (37.43) was reflected by genotype Nagpur Seedless which was followed by Clone-11, CRS-4 and Kinnow. The maximum mean juice redness and greenness value (8.83) was recorded in genotype CRS-4 and it was significantly higher than all other genotypes except Clone11 and W. Murcott which recorded 8.20 and 7.83 values of juice redness and greenness, respectively. The mean juice blueness and yellowness value of different mandarin genotypes ranges from 20.94 to 29.19 with average value of 24.59 . The maximum mean juice blueness and yellowness value (29.19) was recorded in genotype Nova followed by Coorg (26.84) and N-43 (26.70) and it was significantly higher than all other genotypes. Maximum mean value of juice CCI of different mandarin genotypes ranged from 5.69 to 9.78 with average value of 7.59 . The mean value of juice CCI was maximum (9.78) in Daisy which was statistically at par with W. Murcott (9.68), CRS-4 (9.14), Clone-11(9.03) and Kinnow (8.86) and it was significantly higher than all other genotypes under study.

Fig.1 Dendrogram illustrating genetic relationship among 19 mandarin genotypes generated by UPGMA tree analysis based on citrus color index of fruit epicarp, pulp and juice

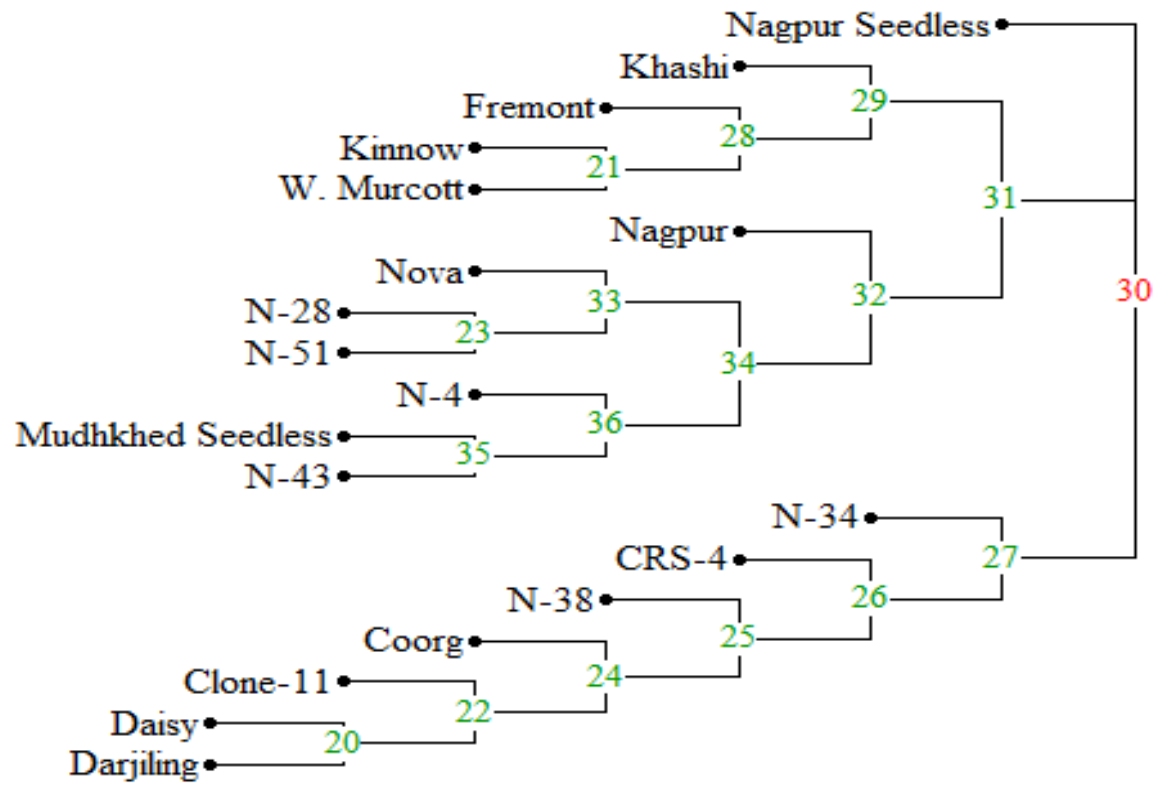


Table.1 Fruit epicarp colour reflectance characters in different mandarin genotypes

\begin{tabular}{|c|c|c|c|c|c|c|c|c|c|c|c|c|}
\hline \multirow[t]{2}{*}{ Genotypes } & \multicolumn{3}{|c|}{$\begin{array}{c}\text { Epicarp lightness } \\
\text { (L) }\end{array}$} & \multicolumn{3}{|c|}{$\begin{array}{l}\text { Epicarp redness and } \\
\text { greenness (a) }\end{array}$} & \multicolumn{3}{|c|}{$\begin{array}{c}\text { Epicarp blueness and } \\
\text { yellowness (b) }\end{array}$} & \multicolumn{3}{|c|}{$\begin{array}{l}\text { Epicarp CCI (Citrus } \\
\text { Colour Index) }\end{array}$} \\
\hline & 2013 & 2014 & Pooled & 2013 & 2014 & Pooled & 2013 & 2014 & Pooled & 2013 & 2014 & Pooled \\
\hline CRS-4 & $56.22^{\mathrm{e}}$ & $58.61^{\mathrm{abc}}$ & $57.42^{\mathrm{bcd}}$ & $18.44^{\mathrm{fg}}$ & $20.82^{\mathrm{gh}}$ & $19.63^{\text {efg }}$ & $52.09^{\mathrm{cd}}$ & $57.42^{\text {def }}$ & $54.76^{\mathrm{bcde}}$ & $6.31^{\mathrm{def}}$ & $6.18^{\mathrm{efg}}$ & $6.24^{\mathrm{fg}}$ \\
\hline Clone-11 & $58.50^{\text {bcde }}$ & $60.64^{\mathrm{a}}$ & $59.57^{\mathrm{ab}}$ & $19.12^{\text {fg }}$ & $23.80^{\mathrm{ef}}$ & $21.46^{\text {cdef }}$ & $60.27^{\mathrm{a}}$ & $62.08^{\mathrm{ab}}$ & $61.18^{\mathrm{a}}$ & $5.42^{\mathrm{fg}}$ & $6.32^{\mathrm{efg}}$ & $5.87^{\mathrm{g}}$ \\
\hline Coorg & $53.10^{\mathrm{fg}}$ & $58.22^{\mathrm{abcd}}$ & $55.66^{\text {def }}$ & $17.55^{\mathrm{gh}}$ & $26.52^{d}$ & $22.04^{\text {cde }}$ & $52.19^{\mathrm{cd}}$ & $58.48^{\mathrm{cde}}$ & $55.34^{\mathrm{bcd}}$ & $6.35^{\mathrm{def}}$ & $7.79^{\mathrm{c}}$ & $7.07^{\mathrm{def}}$ \\
\hline Daisy & $60.26^{\mathrm{bc}}$ & $58.46^{\mathrm{abcd}}$ & $59.36^{\mathrm{ab}}$ & $27.88^{\mathrm{b}}$ & $32.35^{\mathrm{a}}$ & $30.12^{\mathrm{a}}$ & $58.00^{\mathrm{a}}$ & $62.94^{\mathrm{a}}$ & $60.47^{\mathrm{a}}$ & $7.97^{\mathrm{bc}}$ & $8.79^{b}$ & $8.38^{\mathrm{bc}}$ \\
\hline Darjeeling & $59.70^{\text {bcd }}$ & $56.30^{\text {bcdef }}$ & $58.00^{\text {bcd }}$ & $20.37^{\mathrm{def}}$ & $21.49^{\text {fgh }}$ & $20.93^{\mathrm{def}}$ & $53.39^{\mathrm{c}}$ & $57.48^{\mathrm{def}}$ & $55.44^{\mathrm{bc}}$ & $6.39^{\mathrm{de}}$ & $6.64^{\mathrm{de}}$ & $6.51^{\text {efg }}$ \\
\hline Fremont & $57.77^{\text {cde }}$ & $54.98^{\text {cdefg }}$ & $56.38^{\text {cde }}$ & $23.43^{\mathrm{c}}$ & $29.28^{\mathrm{bc}}$ & $26.35^{b}$ & $49.36^{\mathrm{de}}$ & $51.12^{\mathrm{ij}}$ & $50.24^{\text {efg }}$ & $8.21^{b}$ & $10.42^{\mathrm{a}}$ & $9.32^{b}$ \\
\hline Khasi & $57.31^{\mathrm{de}}$ & $56.96^{\text {abcde }}$ & $57.14^{\mathrm{bcd}}$ & $19.19^{\mathrm{fg}}$ & $27.17^{\mathrm{cd}}$ & $23.18^{\mathrm{cd}}$ & $50.59^{\text {cde }}$ & $52.41^{\mathrm{hi}}$ & $51.50^{\text {cdef }}$ & $6.67^{\mathrm{de}}$ & $9.12^{b}$ & $7.89^{\mathrm{cd}}$ \\
\hline Kinnow & $63.10^{\mathrm{a}}$ & $60.26^{\mathrm{a}}$ & $61.68^{\mathrm{a}}$ & $30.87^{\mathrm{a}}$ & $29.62^{b c}$ & $30.25^{\mathrm{a}}$ & $60.29^{\mathrm{a}}$ & $54.88^{\text {fgh }}$ & $57.59^{\mathrm{ab}}$ & $8.11^{b}$ & $8.97^{b}$ & $8.54^{\mathrm{bc}}$ \\
\hline Mudhkhed Seedless & $61.10^{\mathrm{ab}}$ & $58.49^{\mathrm{abcd}}$ & $59.79^{\mathrm{ab}}$ & $19.78^{\text {efg }}$ & $16.98^{\mathrm{ij}}$ & $18.38^{\text {fgh }}$ & $41.13^{h}$ & $59.66^{\text {bcd }}$ & $50.39^{\text {efg }}$ & $7.87^{b c}$ & $4.86^{\text {hi }}$ & $6.37^{\mathrm{fg}}$ \\
\hline $\mathrm{N}-4$ & $57.92^{\text {cde }}$ & $59.50^{\mathrm{ab}}$ & $58.71^{\mathrm{bc}}$ & $21.81^{\text {cde }}$ & $22.65^{\text {efg }}$ & $22.23^{\text {cde }}$ & $57.67^{\mathrm{ab}}$ & $59.16^{\text {bcd }}$ & $58.49^{\mathrm{ab}}$ & $6.52^{\mathrm{de}}$ & $6.43^{\text {def }}$ & $6.47^{\mathrm{efg}}$ \\
\hline $\mathrm{N}-28$ & $47.83^{\mathrm{h}}$ & $53.45^{\mathrm{efg}}$ & $50.64^{\mathrm{g}}$ & $15.20^{\mathrm{i}}$ & $13.47^{1}$ & $14.34^{\mathrm{i}}$ & $47.69^{\mathrm{ef}}$ & $46.14^{\mathrm{k}}$ & $46.92^{\mathrm{fg}}$ & $6.70^{\mathrm{de}}$ & $5.46^{\mathrm{gh}}$ & $6.08^{\mathrm{fg}}$ \\
\hline $\mathrm{N}-34$ & $51.93^{\mathrm{fg}}$ & $53.92^{\text {efg }}$ & $52.92^{\mathrm{fg}}$ & $15.54^{\text {hi }}$ & $16.16^{\mathrm{jk}}$ & $15.85^{\mathrm{hi}}$ & $48.17^{\mathrm{ef}}$ & $53.45^{\text {ghi }}$ & $50.81^{\text {cdefg }}$ & $6.19^{\text {def }}$ & $5.61^{\text {fgh }}$ & $5.90^{\mathrm{g}}$ \\
\hline $\mathrm{N}-38$ & $50.41^{\mathrm{gh}}$ & $53.23^{\mathrm{fg}}$ & $51.82^{\mathrm{g}}$ & $14.68^{\mathrm{i}}$ & $16.87^{\mathrm{ij}}$ & $15.78^{\text {hi }}$ & $43.88^{\mathrm{gh}}$ & $48.43^{\mathrm{jk}}$ & $46.15^{\mathrm{g}}$ & $6.65^{\mathrm{de}}$ & $6.54^{\mathrm{de}}$ & $6.60^{\mathrm{efg}}$ \\
\hline $\mathrm{N}-43$ & $52.13^{\mathrm{fg}}$ & $54.88^{\text {defg }}$ & $53.51^{\mathrm{efg}}$ & $15.77^{\mathrm{hi}}$ & $18.87^{\mathrm{hi}}$ & $17.32^{\text {ghi }}$ & $50.46^{\mathrm{cde}}$ & $48.68^{\mathrm{jk}}$ & $49.57^{\mathrm{fg}}$ & $5.99^{\mathrm{ef}}$ & $7.06^{\mathrm{cde}}$ & $6.53^{\mathrm{efg}}$ \\
\hline $\mathrm{N}-51$ & $57.57^{\mathrm{de}}$ & $60.17^{\mathrm{a}}$ & $58.87^{\mathrm{abc}}$ & $14.74^{\mathrm{i}}$ & $14.39^{\mathrm{jkl}}$ & $14.57^{\mathrm{i}}$ & $52.66^{\mathrm{cd}}$ & $57.67^{\mathrm{def}}$ & $55.17^{\mathrm{bcd}}$ & $4.88^{\mathrm{g}}$ & $4.14^{i}$ & $4.51^{\mathrm{h}}$ \\
\hline Nagpur Seedless & $63.46^{\mathrm{a}}$ & $54.09^{\mathrm{efg}}$ & $58.78^{\mathrm{abc}}$ & $24.06^{\mathrm{c}}$ & $14.17^{\mathrm{kl}}$ & $19.12^{\text {efg }}$ & $47.19^{\text {efg }}$ & $51.76^{\mathrm{i}}$ & $49.48^{\mathrm{fg}}$ & $8.06^{b}$ & $5.06^{\mathrm{h}}$ & $6.56^{\mathrm{efg}}$ \\
\hline Nagpur & $57.71^{\text {cde }}$ & $56.39^{\text {bcdef }}$ & $57.05^{\mathrm{bcd}}$ & $23.77^{\mathrm{c}}$ & $25.02^{\mathrm{de}}$ & $24.40^{\mathrm{bc}}$ & $52.54^{\mathrm{cd}}$ & $61.44^{\mathrm{abc}}$ & $56.99^{\mathrm{ab}}$ & $7.85^{\mathrm{bc}}$ & $7.27^{\mathrm{cd}}$ & $7.56^{\text {cde }}$ \\
\hline Nova & $57.93^{\text {cde }}$ & $59.63^{\mathrm{ab}}$ & $58.78^{\mathrm{abc}}$ & $22.10^{\mathrm{cd}}$ & $20.59^{\text {gh }}$ & $21.35^{\text {cdef }}$ & $54.00^{\mathrm{bc}}$ & $61.92^{\mathrm{ab}}$ & $57.96^{\mathrm{ab}}$ & $7.10^{\mathrm{cd}}$ & $5.60^{\text {fgh }}$ & $6.35^{\mathrm{fg}}$ \\
\hline W. Murcott & $53.33^{f}$ & $52.57^{\mathrm{g}}$ & $52.95^{\mathrm{fg}}$ & $31.69^{\mathrm{a}}$ & $31.46^{\mathrm{ab}}$ & $31.58^{\mathrm{a}}$ & $45.24^{\mathrm{fg}}$ & $56.07^{\text {efg }}$ & $50.65^{\text {defg }}$ & $13.14^{\mathrm{a}}$ & $10.68^{\mathrm{a}}$ & $11.91^{\mathrm{a}}$ \\
\hline Mean & 56.70 & 56.88 & 56.79 & 20.84 & 22.19 & 21.52 & 51.41 & 55.85 & 53.63 & 7.18 & 7.00 & 7.09 \\
\hline LSD $(p \leq 0.05)$ & 2.69 & 3.73 & 2.96 & 2.28 & 2.66 & 3.12 & 3.69 & 2.99 & 4.69 & 0.93 & 0.88 & 1.12 \\
\hline $\mathrm{CV}$ & 2.87 & 3.97 & 4.56 & 6.63 & 7.26 & 12.67 & 4.35 & 3.24 & 7.64 & 7.91 & 7.62 & 13.83 \\
\hline
\end{tabular}

Different alphabets show significant difference and same alphabets show non-significant difference among genotypes 
Table.2 Fruit pulp colour reflectance characters in different mandarin genotypes

\begin{tabular}{|c|c|c|c|c|c|c|c|c|c|c|c|c|}
\hline \multirow[t]{2}{*}{ Genotypes } & \multicolumn{3}{|c|}{$\begin{array}{l}\text { Pulp lightness } \\
\text { (L) }\end{array}$} & \multicolumn{3}{|c|}{$\begin{array}{c}\text { Pulp redness and } \\
\text { greenness (a) }\end{array}$} & \multicolumn{3}{|c|}{$\begin{array}{c}\text { Pulp blueness and } \\
\text { yellowness (b) }\end{array}$} & \multicolumn{3}{|c|}{$\begin{array}{c}\text { Pulp CCI (Citrus } \\
\text { Colour Index) }\end{array}$} \\
\hline & 2013 & 2014 & Pooled & 2013 & 2014 & Pooled & 2013 & 2014 & Pooled & 2013 & 2014 & Pooled \\
\hline CRS-4 & $56.46^{\mathrm{bc}}$ & $50.53^{\mathrm{ef}}$ & $53.50^{\mathrm{abcd}}$ & $6.31^{\mathrm{ghi}}$ & $8.63^{b c d}$ & $7.47^{\text {defg }}$ & $21.09^{\mathrm{fg}}$ & $20.40^{\text {defg }}$ & $20.75^{\mathrm{ef}}$ & $5.30^{\text {ghijk }}$ & $8.38^{\mathrm{b}}$ & $6.84^{\text {bcdef }}$ \\
\hline Clone-11 & $49.12^{\mathrm{ef}}$ & $53.24^{\text {cde }}$ & $51.18^{\text {bcde }}$ & $9.03^{b c}$ & $9.69^{\mathrm{ab}}$ & $9.36^{\mathrm{bc}}$ & $23.41^{\mathrm{def}}$ & $21.31^{\mathrm{def}}$ & $22.36^{\text {cde }}$ & $7.86^{\mathrm{cd}}$ & $8.51^{b}$ & $8.18^{b}$ \\
\hline Coorg & $43.18^{\mathrm{i}}$ & $59.41^{\mathrm{a}}$ & $51.30^{\text {bcde }}$ & $8.91^{\text {bcd }}$ & $6.79^{\mathrm{fg}}$ & $7.85^{\text {def }}$ & $21.98^{\text {efg }}$ & $19.41^{\text {efgh }}$ & $20.70^{\mathrm{ef}}$ & $9.45^{b}$ & $5.91^{\mathrm{cde}}$ & $7.68^{\mathrm{bcd}}$ \\
\hline Daisy & $30.92^{\mathrm{k}}$ & $55.50^{\mathrm{bcd}}$ & $43.21^{\mathrm{fg}}$ & $12.84^{\mathrm{a}}$ & $8.75^{\text {bcd }}$ & $10.79^{\mathrm{a}}$ & $30.14^{\mathrm{a}}$ & $18.14^{\text {fgh }}$ & $24.14^{\text {cde }}$ & $13.79^{\mathrm{a}}$ & $8.80 \mathrm{~b}$ & $11.30^{\mathrm{a}}$ \\
\hline Darjeeling & $40.04^{j}$ & $41.64^{\mathrm{h}}$ & $40.84^{\mathrm{g}}$ & $9.47^{b}$ & $10.51^{\mathrm{a}}$ & $9.98^{\mathrm{ab}}$ & $26.44^{b c}$ & $21.35^{\mathrm{def}}$ & $23.89^{\text {cde }}$ & $8.98^{\mathrm{bc}}$ & $12.03^{\mathrm{a}}$ & $10.51^{\mathrm{a}}$ \\
\hline Fremont & $46.46^{\mathrm{gh}}$ & $56.41^{\mathrm{abc}}$ & $51.43^{\text {bcde }}$ & $7.99^{\text {cde }}$ & $8.88^{\mathrm{bc}}$ & $8.44^{\text {cd }}$ & $29.10^{\mathrm{ab}}$ & $28.20^{\mathrm{ab}}$ & $28.69^{\mathrm{ab}}$ & $5.96^{\text {efghi }}$ & $5.61^{\text {cdef }}$ & $5.79^{\text {fgh }}$ \\
\hline Khasi & $51.62^{\mathrm{de}}$ & $59.48^{\mathrm{a}}$ & $55.55^{\mathrm{abc}}$ & $7.36^{\mathrm{efg}}$ & $7.70^{\text {def }}$ & $7.53^{\text {defg }}$ & $28.87^{\mathrm{ab}}$ & $22.86^{\mathrm{cd}}$ & $25.87^{\mathrm{abc}}$ & $4.93^{\text {hijk }}$ & $5.68^{\mathrm{cde}}$ & $5.31^{\text {fgh }}$ \\
\hline Kinnow & $44.31^{\mathrm{hi}}$ & $53.59^{\text {cde }}$ & $48.95^{\mathrm{def}}$ & $7.54^{\text {defg }}$ & $9.67^{\mathrm{ab}}$ & $8.61^{\mathrm{cd}}$ & $30.92^{\mathrm{a}}$ & $27.23^{\mathrm{ab}}$ & $29.08^{\mathrm{a}}$ & $5.55^{\text {fghi }}$ & $6.60^{\text {cde }}$ & $6.08^{\mathrm{defg}}$ \\
\hline Mudhkhed Seedless & $48.07^{\mathrm{fg}}$ & $52.14^{\text {def }}$ & $50.10^{\text {cde }}$ & $8.02^{\text {cde }}$ & $6.14^{\mathrm{gh}}$ & $7.08^{\text {efgh }}$ & $26.64^{\mathrm{bc}}$ & $21.91^{\text {cde }}$ & $24.28^{\text {cde }}$ & $6.27^{\text {efgh }}$ & $5.52^{\text {cdef }}$ & $5.90^{\text {fgh }}$ \\
\hline $\mathrm{N}-4$ & $55.73^{\mathrm{c}}$ & $50.93^{\mathrm{ef}}$ & $53.33^{\mathrm{abcd}}$ & $6.55^{\text {fghi }}$ & $7.51^{\mathrm{ef}}$ & $7.03^{\text {efgh }}$ & $21.67^{\text {efg }}$ & $21.98^{\text {cde }}$ & $21.83^{\mathrm{def}}$ & $5.42^{\text {ghij }}$ & $6.72^{\mathrm{cd}}$ & $6.07^{\text {defg }}$ \\
\hline $\mathrm{N}-28$ & $56.69^{\mathrm{bc}}$ & $59.09^{\mathrm{ab}}$ & $57.89^{\mathrm{a}}$ & $5.64^{\mathrm{hi}}$ & $8.21^{\text {cde }}$ & $6.93^{\text {fghi }}$ & $25.42^{\mathrm{cd}}$ & $26.47^{\mathrm{ab}}$ & $25.95^{\mathrm{abc}}$ & $3.94^{\mathrm{jk}}$ & $5.27^{\mathrm{def}}$ & $4.60^{\mathrm{gh}}$ \\
\hline $\mathrm{N}-34$ & $47.68^{\mathrm{fg}}$ & $46.19^{\mathrm{g}}$ & $46.94^{\mathrm{ef}}$ & $5.58^{\text {hi }}$ & $5.78^{\text {ghi }}$ & $5.68^{\mathrm{ijk}}$ & $16.40^{\mathrm{hi}}$ & $14.45^{\mathrm{i}}$ & $15.43 \mathrm{~g}$ & $7.06^{\mathrm{def}}$ & $8.67^{\mathrm{b}}$ & $7.87^{\mathrm{bc}}$ \\
\hline $\mathrm{N}-38$ & $51.02^{\mathrm{de}}$ & $52.49^{\mathrm{def}}$ & $51.75^{\text {bcde }}$ & $6.97^{\mathrm{efgh}}$ & $5.24^{\mathrm{hi}}$ & $6.10^{\text {hijk }}$ & $15.18^{\mathrm{i}}$ & $16.36^{\mathrm{hi}}$ & $15.77^{\mathrm{g}}$ & $9.03^{b c}$ & $6.10^{\text {cde }}$ & $7.56^{\text {bcde }}$ \\
\hline $\mathrm{N}-43$ & $52.20^{\mathrm{d}}$ & $53.98^{\mathrm{cde}}$ & $53.09^{\mathrm{abcd}}$ & $6.18^{\mathrm{ghi}}$ & $5.00^{i}$ & $5.59^{\mathrm{jk}}$ & $19.22^{\mathrm{gh}}$ & $18.06^{\text {fgh }}$ & $18.64^{\mathrm{fg}}$ & $6.48^{\text {defg }}$ & $5.17^{\mathrm{ef}}$ & $5.83^{\text {fgh }}$ \\
\hline $\mathrm{N}-51$ & $50.91^{\mathrm{de}}$ & $54.07^{\text {cde }}$ & $52.49^{\text {abcde }}$ & $5.37^{i}$ & $4.90^{\mathrm{i}}$ & $5.14^{\mathrm{k}}$ & $23.87^{\text {cdef }}$ & $22.23^{\text {cde }}$ & $23.05^{\text {cde }}$ & $4.43^{\mathrm{ijk}}$ & $4.13^{\mathrm{f}}$ & $4.28^{h}$ \\
\hline Nagpur Seedless & $44.54^{\mathrm{hi}}$ & $54.60^{\mathrm{cd}}$ & $49.57^{\mathrm{de}}$ & $7.84^{\text {cdef }}$ & $5.64^{\mathrm{hi}}$ & $6.74^{\text {fghij }}$ & $24.41^{\text {cde }}$ & $17.89^{\mathrm{gh}}$ & $21.15^{\mathrm{ef}}$ & $7.30^{\mathrm{de}}$ & $5.81^{\mathrm{cde}}$ & $6.60^{\text {cdef }}$ \\
\hline Nagpur & $60.11^{\mathrm{a}}$ & $49.59^{\mathrm{fg}}$ & $54.85^{\mathrm{abcd}}$ & $7.08^{\mathrm{efg}}$ & $5.72^{\text {hi }}$ & $6.40^{\text {ghijk }}$ & $30.85^{\mathrm{a}}$ & $19.57^{\text {defgh }}$ & $25.21^{\mathrm{bcd}}$ & $3.87^{\mathrm{k}}$ & $5.91^{\mathrm{cde}}$ & $4.89^{\text {gh }}$ \\
\hline Nova & $52.59^{d}$ & $56.86^{\mathrm{abc}}$ & $54.73^{\mathrm{abcd}}$ & $8.23^{\text {bcde }}$ & $8.47^{\text {cde }}$ & $8.35^{\mathrm{cde}}$ & $28.69^{\mathrm{ab}}$ & $29.25^{\mathrm{a}}$ & $28.97^{\mathrm{a}}$ & $5.50^{\text {ghi }}$ & $5.11^{\mathrm{ef}}$ & $5.30^{\mathrm{fgh}}$ \\
\hline W. Murcott & $58.94^{\mathrm{ab}}$ & $53.38^{\text {cde }}$ & $56.16^{\mathrm{ab}}$ & $8.28^{\text {bcde }}$ & $9.04^{b c}$ & $8.66^{\mathrm{bcd}}$ & $26.64^{\mathrm{bc}}$ & $24.96^{\mathrm{bc}}$ & $25.80^{\mathrm{abc}}$ & $5.28^{\text {ghijk }}$ & $6.80^{c}$ & $6.04^{\text {efg }}$ \\
\hline Mean & 49.50 & 53.32 & 51.41 & 7.64 & 7.49 & 7.56 & 24.79 & 21.69 & 23.24 & 6.65 & 6.67 & 6.66 \\
\hline LSD (p $\leq 0.05)$ & 2.64 & 3.63 & 5.90 & 1.42 & 1.07 & 1.33 & 2.97 & 3.35 & 3.59 & 1.53 & 1.49 & 1.62 \\
\hline $\mathrm{CV}$ & 3.23 & 4.13 & 10.02 & 11.26 & 8.69 & 15.41 & 7.27 & 9.37 & 13.51 & 14.00 & 13.57 & 21.25 \\
\hline
\end{tabular}

Different alphabets show significant difference and same alphabets show non-significant difference among genotypes 
Table.3 Fruit juice colour reflectance characters in different mandarin genotypes

\begin{tabular}{|c|c|c|c|c|c|c|c|c|c|c|c|c|}
\hline \multirow[t]{2}{*}{ Genotypes } & \multicolumn{3}{|c|}{$\begin{array}{l}\text { Juice lightness } \\
\text { (L) }\end{array}$} & \multicolumn{3}{|c|}{$\begin{array}{c}\text { Juice redness and } \\
\text { greenness (a) }\end{array}$} & \multicolumn{3}{|c|}{$\begin{array}{c}\text { Juice blueness and } \\
\text { yellowness (b) }\end{array}$} & \multicolumn{3}{|c|}{$\begin{array}{c}\text { Juice CCI (Citrus } \\
\text { Colour Index) }\end{array}$} \\
\hline & 2013 & 2014 & Pooled & 2013 & 2014 & Pooled & 2013 & 2014 & Pooled & 2013 & 2014 & Pooled \\
\hline CRS-4 & $36.10^{\text {abcdef }}$ & $36.31^{\mathrm{a}}$ & $36.21^{\mathrm{ab}}$ & $8.80^{\mathrm{a}}$ & $8.86^{\mathrm{ab}}$ & $8.83^{\mathrm{a}}$ & $26.30^{\text {bcd }}$ & $27.10^{\mathrm{ab}}$ & $26.70^{\text {bcd }}$ & $9.28^{\mathrm{a}}$ & $9.01^{\mathrm{abcd}}$ & $9.14^{\mathrm{ab}}$ \\
\hline Clone-11 & $38.16^{\mathrm{abc}}$ & $36.42^{\mathrm{a}}$ & $37.29^{\mathrm{a}}$ & $8.85^{\mathrm{a}}$ & $7.54^{\mathrm{cd}}$ & $8.20^{\mathrm{ab}}$ & $24.85^{\text {bcdefg }}$ & $24.00^{\text {cdef }}$ & $24.42^{\text {defgh }}$ & $9.36^{\mathrm{a}}$ & $8.70^{\text {abcde }}$ & $9.03^{\mathrm{abc}}$ \\
\hline Coorg & $34.85^{\text {defg }}$ & $33.79^{\mathrm{ab}}$ & $34.32^{\mathrm{bc}}$ & $5.54^{\text {fgh }}$ & $9.21^{\mathrm{a}}$ & $7.38^{\text {bcde }}$ & $26.77^{\mathrm{abc}}$ & $26.91^{\mathrm{abc}}$ & $26.84^{b}$ & $5.95^{\mathrm{ef}}$ & $10.17^{\mathrm{a}}$ & $8.06^{\text {bcde }}$ \\
\hline Daisy & $35.49^{\text {cdefg }}$ & $36.63^{\mathrm{a}}$ & $36.06^{\mathrm{ab}}$ & $6.69^{\text {cde }}$ & $7.94^{\text {abcd }}$ & $7.32^{\text {bcde }}$ & $20.58^{\mathrm{ij}}$ & $21.31^{\mathrm{fg}}$ & $20.94^{j}$ & $9.27^{\mathrm{a}}$ & $10.29^{\mathrm{a}}$ & $9.78^{\mathrm{a}}$ \\
\hline Darjeeling & $37.11^{\mathrm{abcd}}$ & $33.88^{\mathrm{ab}}$ & $35.50^{\mathrm{abc}}$ & $4.99^{\mathrm{hij}}$ & $8.21^{\mathrm{abcd}}$ & $6.60^{\text {defg }}$ & $24.15^{\text {cdefgh }}$ & $25.91^{\text {bcd }}$ & $25.03^{\text {bcdef }}$ & $5.58^{f}$ & $9.50^{\mathrm{abc}}$ & $7.55^{\text {defg }}$ \\
\hline Fremont & $33.34^{\mathrm{fg}}$ & $36.60^{\mathrm{a}}$ & $34.97^{\mathrm{abc}}$ & $5.36^{\text {ghi }}$ & $4.95^{\text {ghi }}$ & $5.16^{\text {hij }}$ & $26.10^{\text {bcde }}$ & $25.86^{\text {bcd }}$ & $25.98^{\text {bcde }}$ & $6.16^{\text {def }}$ & $5.21^{\mathrm{h}}$ & $5.69^{h}$ \\
\hline Khasi & $35.83^{\text {bcdefg }}$ & $33.68^{\mathrm{ab}}$ & $34.76^{\mathrm{abc}}$ & $5.78^{\text {efgh }}$ & $7.34^{\text {cdef }}$ & $6.57^{\text {defg }}$ & $23.44^{\text {defghi }}$ & $26.58^{\mathrm{abcd}}$ & $25.01^{\text {bcdefg }}$ & $6.94^{\text {cdef }}$ & $8.25^{\text {bcdef }}$ & $7.59^{\mathrm{def}}$ \\
\hline Kinnow & $35.43^{\text {cdefg }}$ & $36.76^{\mathrm{a}}$ & $36.10^{\mathrm{ab}}$ & $6.70^{\text {cde }}$ & $8.36^{\mathrm{abc}}$ & $7.53^{\mathrm{bcd}}$ & $22.12^{\text {ghij }}$ & $25.07^{\text {bcde }}$ & $23.60^{\text {fghi }}$ & $8.53^{\mathrm{ab}}$ & $9.19^{\mathrm{abcd}}$ & $8.86^{\text {abcd }}$ \\
\hline Mudhkhed Seedless & $36.50^{\text {abcde }}$ & $33.35^{\mathrm{ab}}$ & $34.93^{\mathrm{abc}}$ & $4.43^{j}$ & $6.23^{\text {efg }}$ & $5.33^{\text {hij }}$ & $20.04^{j}$ & $24.91^{\text {bcde }}$ & $22.47^{\text {hij }}$ & $6.03^{\mathrm{ef}}$ & $7.52^{\text {defg }}$ & $6.78^{\text {efgh }}$ \\
\hline $\mathrm{N}-4$ & $33.19^{\mathrm{gh}}$ & $33.08^{\mathrm{ab}}$ & $33.14^{\mathrm{cd}}$ & $4.61^{\mathrm{ij}}$ & $4.73^{i}$ & $4.67^{j}$ & $23.72^{\text {defgh }}$ & $20.44^{\mathrm{g}}$ & $22.08^{\mathrm{ij}}$ & $5.90^{\mathrm{ef}}$ & $6.98^{\mathrm{efg}}$ & $6.44^{\text {fgh }}$ \\
\hline $\mathrm{N}-28$ & $36.75^{\text {abcde }}$ & $35.13^{\mathrm{ab}}$ & $35.94^{\mathrm{ab}}$ & $6.07^{\mathrm{efg}}$ & $5.64^{\text {ghi }}$ & $5.86^{\text {fghi }}$ & $27.59^{\mathrm{ab}}$ & $25.53^{\text {bcd }}$ & $26.56^{\mathrm{bcd}}$ & $6.06^{\mathrm{def}}$ & $6.25^{\mathrm{gh}}$ & $6.16^{\mathrm{gh}}$ \\
\hline $\mathrm{N}-34$ & $35.65^{\text {bcdefg }}$ & $35.60^{\mathrm{ab}}$ & $35.63^{\mathrm{abc}}$ & $5.82^{\text {efgh }}$ & $5.61^{\text {ghi }}$ & $5.71^{\text {ghij }}$ & $25.03^{\text {bcdefg }}$ & $24.43^{\text {bcde }}$ & $24.73^{\text {bcdefgh }}$ & $6.52^{\text {cdef }}$ & $6.47^{\text {gh }}$ & $6.49^{\mathrm{gh}}$ \\
\hline $\mathrm{N}-38$ & $38.83^{\mathrm{a}}$ & $33.01^{\mathrm{ab}}$ & $35.92^{\mathrm{ab}}$ & $7.53^{\mathrm{bc}}$ & $6.14^{\text {fgh }}$ & $6.84^{\text {cdefg }}$ & $25.05^{\text {bcdef }}$ & $23.90^{\text {cdef }}$ & $24.47^{\text {cdefgh }}$ & $7.77^{\mathrm{bc}}$ & $7.79^{\text {cdefg }}$ & $7.78^{\text {bcdef }}$ \\
\hline $\mathrm{N}-43$ & $36.96^{\mathrm{abcde}}$ & $32.95^{\mathrm{ab}}$ & $34.96^{\mathrm{abc}}$ & $6.41^{\mathrm{def}}$ & $6.04^{\text {fgh }}$ & $6.23^{\mathrm{efgh}}$ & $27.09^{\mathrm{ab}}$ & $26.33^{\mathrm{abcd}}$ & $26.71^{\mathrm{bc}}$ & $6.43^{\text {cdef }}$ & $6.99^{\mathrm{efg}}$ & $6.71^{\text {efgh }}$ \\
\hline $\mathrm{N}-51$ & $30.47^{\mathrm{h}}$ & $31.52^{b}$ & $31.00^{\mathrm{d}}$ & $5.10^{\mathrm{hij}}$ & $4.90^{\mathrm{hi}}$ & $5.00^{\mathrm{ij}}$ & $22.20^{\text {fghij }}$ & $25.24^{\text {bcde }}$ & $23.72^{\text {efghi }}$ & $7.54^{\mathrm{bcd}}$ & $6.19^{\text {gh }}$ & $6.87^{\text {efgh }}$ \\
\hline Nagpur Seedless & $38.45^{\mathrm{ab}}$ & $36.40^{\mathrm{a}}$ & $37.43^{\mathrm{a}}$ & $5.77^{\text {fgh }}$ & $7.47^{\text {cde }}$ & $6.62^{\text {defg }}$ & $22.37^{\text {fghij }}$ & $23.59^{\text {def }}$ & $22.98^{\text {fghij }}$ & $6.70^{\text {cdef }}$ & $8.73^{\text {abcd }}$ & $7.72^{\text {cdef }}$ \\
\hline Nagpur & $35.53^{\text {cdefg }}$ & $33.23^{\mathrm{ab}}$ & $34.38^{\mathrm{bc}}$ & $4.59^{\mathrm{ij}}$ & $6.14^{\mathrm{fgh}}$ & $5.36^{\text {hij }}$ & $21.42^{\mathrm{ij}}$ & $24.48^{\text {bcde }}$ & $22.95^{\text {fghij }}$ & $6.09^{\mathrm{def}}$ & $7.60^{\text {defg }}$ & $6.85^{\text {efgh }}$ \\
\hline Nova & $34.18^{\text {efg }}$ & $34.54^{\mathrm{ab}}$ & $34.36^{\mathrm{bc}}$ & $7.08^{\mathrm{bcd}}$ & $6.98^{\mathrm{def}}$ & $7.03^{\text {bcdef }}$ & $29.45^{\mathrm{a}}$ & $28.94^{\mathrm{a}}$ & $29.19^{\mathrm{a}}$ & $7.07^{\text {bcde }}$ & $6.97^{\mathrm{fg}}$ & $7.02^{\text {efgh }}$ \\
\hline W. Murcott & $36.04^{\text {abcdef }}$ & $35.45^{\mathrm{ab}}$ & $35.74^{\mathrm{abc}}$ & $7.80^{b}$ & $7.87^{\text {bcd }}$ & $7.83^{\mathrm{abc}}$ & $23.23^{\text {efghi }}$ & $22.27^{\mathrm{efg}}$ & $22.75^{\text {ghij }}$ & $9.42^{\mathrm{a}}$ & $9.94^{\mathrm{ab}}$ & $9.68^{\mathrm{a}}$ \\
\hline Mean & 35.73 & 34.65 & 35.19 & 6.21 & 6.85 & 6.53 & 24.29 & 24.88 & 24.59 & 7.19 & 7.99 & 7.59 \\
\hline LSD (p $\leq 0.05)$ & 2.82 & 4.34 & 2.71 & 0.91 & 1.30 & 1.17 & 2.92 & 3.02 & 2.27 & 1.49 & 1.72 & 1.42 \\
\hline $\mathrm{CV}$ & 4.78 & 7.58 & 6.73 & 8.97 & 11.50 & 15.75 & 7.29 & 7.35 & 8.08 & 12.56 & 13.03 & 16.40 \\
\hline
\end{tabular}

Different alphabets show significant difference and same alphabets show non-significant difference among genotypes 
The high positive value of CCI indicates the red orange colour of the fruit juice. Daisy and W. Murcott, CRS-4, Clone-11 and Kinnow fruits having higher CCI values are promising genotypes in terms of their juice colour.

\section{Clustering of genotypes}

The dendrogram generated through unweighted pair group method with arithmetic mean (UPGMA) analysis based on the fruit color characteristics clearly divided the nineteen mandarin genotypes into three major groups (Fig. 1). Cluster-1 included only single genotype viz. Nagpur Seedless. The cluster-2 is further divided into two sub clusters namely cluster-2A and cluster-2B. In cluster-2A, four genotypes i.e. Khasi, Fremont, Kinnow and W. Murcott were grouped whereas, in cluster2B, seven genotypes namely Nagpur, Nova, N-28, N-51, N-4, Mudhkhed Seedless and N43 were included. In cluster-3, seven genotypes viz. N-434, CRS-4, N-38, Coorg, Clone-11, Daisy and Darjiling were grouped. The study clearly showed that genotypes were clustered across the groups based on their quantitative colorimetric values without clear evidence of their geographical distribution. It was also observed that hybrids like Kinnow, Fremont, W. Murcott and Daisy were clearly differentiated from the other mandarin selection on the basis of their chromicity scores of fruit epicarp, pulp and juice.

The results are promising and demonstrate the feasibility of using hunter lab to inspect the fruit colour during harvesting in different mandarin genotypes. Most of the mandarin hybrids are differentiated from the mandarin selections on the basis of their chromicity scores which indicates deep red orange colour of the fruit epicarp, pulp and juice. These variation, no doubt indicates the desirability of the breeder to develop deep red orange colour of the fruit in mandarin hybrids. The literature also supported the findings that breeder generally take one pigmented parent in conventional fruit breeding programme (Deng and $\mathrm{Xu}, 2011$ ) and the major focus of the breeder to develop coloured varieties (Abouzar and Nafiseh, 2016). Fruit colour is a very complex trait which is controlled by chemical, biochemical and physicochemical mechanism (Sinha et al., 2012) and several other factors like fruit maturity, tree nutrition, rootstock, agronomic practices and biotic and abiotic stresses (Ladanyia, 2010). Under subtropical conditions, during NovemberDecember, the chlorophyll present in the peel are degraded, carotenes are freshly synthesized (Sinclair, 1984) which imparts yellow colour to the fruit. High variation in fruit colorimetric parameters in all genotypes over the years was due to the air temperature variation and it was greatly influenced by the genetic makeup of the genotype and their parentage. Regulatory gene expression contributes several physiological changes that contribute a lot to the fruit colour development (Torres et al., 2010).

In our experiment, the mid to late season maturing genotypes like W. Murcort, Kinnow, Fremont had higher CCI for epicarp due to the increase in colorimetric coordinate a. The correlation studies of temperature and lemon colour in 'Eureka' and 'Fino' varieties stated that highest ' $a$ ' component of CCI due to fall in temperature in November and December (Manera et al., 2008). Due to lower temperature the 'chlorophyll a' degrades rapidly and carotenoids provide yellow color to fruit epicarp in citrus fruits (Gross, 1991). It was also reported that colour of the peel in lemon fruit begins to change from green to yellow when the minimum temperature was below $15{ }^{\circ} \mathrm{C}$ (Manera et al., 2013). Three different variables $\mathrm{L}, \mathrm{a}$ and $\mathrm{b}$ varied within same genotype in both the years of investigation proved the direct correlation of these variable with temperature, fruit maturity and other physiological processes. More 
importantly, the colorimetric coordinate ' $a$ ' does not depend upon the mean temperature but directly influenced by minimum temperature of the day as an independent variable (Manera et al., 2013).

The mandarin genotypes under study belong to different maturity group and have different geographical origins, thus different chromicity scores describe their colour change time under sub-tropical conditions. The commercial citrus growers have to give certain degreening treatments, depending upon their standard colour index at harvest (Vidal et al., 2013). The study will help the progressive farmers to ensure the uniform quality in term of pulp and flesh colour while packing the fruits in batches. This study will also help the fruit breeder to select the early genotypes on the basis of their colour and may help him to use these genotypes in citrus crop improvement program.

In conclusion, the study demonstrates the feasibility of use of hunter lab to measure chromicity scores before fruit harvesting. Furthermore, it will help to identify the promising parents for citrus improvement programme. W. Murcott, Fremont, Kinnow and Daisy emerged as promising cultivars in terms of their CCI index values and these genotypes should be used in breeding programme for developing deep colored mandarin varieties. The study conclusively proves that chromicity can be used as maturity index for citrus fruits.

\section{References}

Abouzar, A. and Nafiseh, A. M. 2016. The investigation of citrus fruit quality. Popular characteristic and breeding. Acta Universitatis Agriculturae ET Silviculturae Mendelianae Brunensis. 64(3): 725-740.

Artes, F., Minguez, M.I., Hornero, D. 2002.
Analyzing changes in fruit pigments. In: MacDougall DB (Ed.) Color in Food. Improving quality. Woodhead Publishing Limited, Cambridge.

Campbell, B.L., Nelson, R.G., Ebel, C.E., Dozier, W.A., Adrian, J.L. and Hockema, B. R. 2004. Fruit quality characteristics that affect consumer preferences for satsuma mandarins. Hort. Sci. 39(7):1664-1666.

Cubero, S., Molto, E., Gutierrez, A., Aleixos, N., Garcia-Navarrete, O.L., Juste. F. and Blasco, J. 2010. Real-time inspection of fruit on a mobile harvesting platform in field conditions using computer vision. Prog. Agric. Engineering Sci. 6:1-16.

Deng, Z. and $\mathrm{Xu}$, J. 2011. Breeding for fruit quality in citrus. In: Breeding for fruit quality. 349-371.

DOGV. 2006. Diari Oficial de la Comunitat Valenciana. 5346: 30321-30328.

Gardner, J.L. 2007. Comparison of calibration methods for tristimulus colorimeters. $J$. Res. National Institute Standards Tech. 112:129-138.

Gross, J. 1991. Pigments in vegetables. Chlorophylls and carotenoid. Van Nostrand Reinhold, New York.

Haisman, D.R., Clarke, M.W. 1975. The interfacial factor in the heat-induced conversion of chlorophyll to pheophytin in green leaves. J. Sci. Food Agr. 26:1111-1126.

Hunter, Lab. 2008. Application note, 8(9), http://www.hunterlab.com/appnotes/an0 8_96a.pdf. Accessed 16 April.2017.

Hutchings, J.B. 2003. Expectations and the food industry. Kluver Academic/Plenum Publishers, New York.

IPGRI, 1999. Descriptors of Citrus. International Plant Genetic Resource Institute, Rome, Italy. (Available from http:/www.cgiar.org/ipgri/). 
Jenks, M. A. and Bebeli, P. (Eds.) 2011. Breeding for fruit quality. John Wiley \& Sons.

Ladanyia, M. 2010. Citrus fruit: biology, technology and evaluation. San Diego, California, USA: Academic press.

Manera, J., Brotons, J.M., Conesa, A. and Porras, I. 2013. Relationship between air temperature and degreening of lemon (Citrus lemon L. Burm. f.) peel color during maturation. Australian $J$ Crop Sci. 6(6):1051-1058.

Manera, J., Ruiz, G., Fernández, J.C., Conesa, A., Robles, J.M. and Porras, I. 2008. Influencia de la temperatura sobre las coordenadas colorimétricas $\mathrm{L}$, a y b en la evolución Del color externo en los frutos de limón. Levante Agrícola, 47(1):65-70.

Perrier, X., Jacquemoud-Collet, J.P. 2006. DARwin software (online) Available: http:/ /darwin.cirad.fr/darwin.
Sinclair, W.B. 1984. The biochemistry and physiology of the lemon and other citrus fruits. Univ. of California, Oakland.

Sinha, N., Sidhu, J., Barta, J., Wu, J. and Cano, M.P. (Eds.). 2012. Handbook of fruits and fruit processing. John Wiley and Sons.

Torres, D.C., Di'az-Maroto, M.C., Hermosi'n-Gutie'rrez. I., Pe'rez-Coello, M.S. 2010. Effect of freeze-drying and oven drying on volatiles and phenolics composition of grape skin. Anal Chim Acta. 660:177-82.

Vidal, A., Talens, P., Prats-Montalban, J. M., Cubero. S., Albert. F. and Blasco, J. 2013. In-line estimation of the standard colour index of citrus fruits using a computer vision system developed for a mobile platform. Food Bioprocess Technol. 6: 3412-3419.

\section{How to cite this article:}

Gurteg Singh, Phupinder Singh Aulakh and Harinder Singh Rattanpal. 2017. Determination of Maturity and Genetic Diversity in Mandarin (Citrus reticulata Blanco) Genotypes Based on Citrus Colour Index. Int.J.Curr.Microbiol.App.Sci. 6(7): 577-586. doi: https://doi.org/10.20546/ijcmas.2017.607.070 